term paint for šindu; lîru is also a special kind of paint but the qualifying adjective is broken away. At least Clay's suggestion in BE XIV 28 that sindu means "wool", is quite out of the question. šindu cannot have been a very valuable article since it appears as a very common and cheap commodity; in BE XIV 67, Mudamiku receives six mana of šindu for a door, and in no. 74, Etiru-ikbi receives $1 / 3$ (?) of a mana of sindu for a bed. In Strassmaier's, Nebuchadnezzar Texts no. 222, four mana šindu are given to a carpenter; no. 126, sixteen mana šindi to a carpenter and his companions.

Šindu means also "mark on a slave" and in the classical period had the form simtu, Ham. Code 38, 67, which Ungnad, OLZ 1908 Beiheft II 5, connects with simg. Now še-gin is explained by šimtu R. II 7 a $15=\mathrm{V} 39$ e 34 . This compels the assumption that simtu and sindu are the same words and that sindu, paint, and sindu, mark, are likewise the same words, since the Sumerian word šegin equals all of them. 5 g means a mark burned into the Hesh, but since in the Code there is question concerning the changing of a mark, it appears probable that the Babylonians painted the marks on their slaves. Yet it is difficult to explain why they derived the common word for paint, varnish etc. from the root gro, to cut or burn a mark. In ZA III 320, 62 Evett's transliteration does not agree with his text which reads sindu, yet registered by MussArnolt (!) ${ }^{1}$.

In R. II $30 \mathrm{a} 43--47$ five kinds of šibuointments or salves are mentioned, 1. šim guškin, yellow ointment, 2. šim-kas-sî̀-sîg, pale green beer-ointment (?), 3. imi-guškin, yellow paste, 4. šim-sah̆ar (cf. šim-nà-sahar) = ?, 5) šim kas guškin, yellow beer ointment (?); the same list explains lîru in terms identical with 2,4 and 5 above.

Since š́bu is from the root šâpu, rub, polish, the widely used architectural term šs mean polished, varnished surface bence ceiling.

Concerning the ointment egi $\mathrm{HW} 16$ which Delitzsch conjectures to be a loan-word, I can find no new information. Delitzsch's conjecture is supported by the loan-word egizaggiu. guhlu is apparently a Joan-word from

${ }^{1}$ It is tempting to connect šmdu with

paint. In that case the convection of simtu with would have to be given up. gi-hul 1 . For guhlu determined by $i m$, see SAI 6301. Meissner ZA VIII 75, influenced by the old explanation of ladutu, a vessel, explained guhlu as a vessel. didu and sadidu which occur as names of pastes and similar preparations, $R$ V 32 a-c, 26-28, are likewise to be corrected. Whether didu is to be read titu, mud, with Delitzsch is open to question, but sadidu

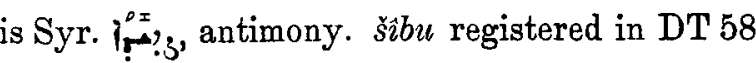
[see CT XVIII 12 insertion after line 88] as an equivalent of nahlaptu urī, is undoubtably the same word. For urî hut, house, see Babyloniaca II 119. The entire list K 169 refers to articles of clothing or adornments, cf. 11. $83 \mathrm{f}$. danitum, gulale, apparels for war, nahlaptu tahā $z i ; 85-88+$ three lines of DT 58 , nahlaptu urī garments worn on the roof of a house; 89-94 nahlaptu bit ahi, garments worn out of doors. In this same list of ornamented robes etc. occurs egizaggı 1. 68 mentioned above as possibly connected with egit, varnish, paste etc. šîbu was therefore, probably a kind of preparation used for coloring the eyes, and face, hence its further application to a luxurious and gay garment. Does the same word occur in Nimrod Epic XI 262, têdikư šipa $\vec{a}$ iddi-ma!

\section{Les dieux Uraš et Išum}

par P. Dhorme.

Le dieu Uraš qui figure dans les textes sous son idéogramme IB, est, avant tout, le dieu des plantations. C'est pourquoi Hammurabi se vante, dans son code, d'avoir étendu les plantations (me-ri-eš-tim) et d'avoir accumulé les tas de blé pour le dieu Uraš (CH, recto, III, 18 ss.). C'est pour cela aussi que ce dieu s'identifie avec le dieu $N i n-i b$ et avec le dieu Nin-gir-su, ce dernier étant le véritable seigneur des plantations, bêt me-riš-ti (šurpî IV, 80). Pourquoi alors ne pas voir dans le nom de Uraš une allusion à cet attribut du dieu? On aurait affaire à une forme urâšu, provenant de erêšu "planter" (d'où mêrištu), comme on a qurâda de qarâdu.

Un autre dieu, portant un nom sémitique, est, selon nous, le dieu $I$-šum. On reconnaît l'accusatif de ce nom dans $I$-šá-am de CT, $\mathrm{XV}$, pl. 6, VIII, 7. On sait aussi qu'il s'agit

1 Zimmern, KAT 649, Ges.-Buhl, 307a, correctly 5. fouud in Arabic, Ethiopic and Aramaic. The word would ger seem to be good Semitic, in which case gi-llul is a loan-word from the Semitic. 
spécialement d'un dieu du feu. Nous sommes donc en présence du mot išu, masculin de išatu "feu". A cette forme $i s ̌ u$ correspond l'hébreu $v$ ș.

\section{MANZAZ ISSUR.}

Von Otto Weber.

In der ersten der von King neuveröffentlichten Chroniken (Br. M. 26472) heisst es Obv. 9-10.

9 a-na mâtu ka-șal-la il-li-k-ma mâtu ka-șal-la a-na tili u kar-me ú-tir

10 ina lib-bi-šu man-za-az iṣ-ṣur th-bूal-lik.

9 Gegen Kașalla zog er und Kaşalla machte er zum Schutt- und Trümmerhaufen;

10 in ihm (dem Lande) zerstörte er den Standort des Vogels.

Diese wörtliche Übersetzung von Z. 10 gibt keinen Sinn, auch nicht die Umschreibung Kings: "he destroyed (the land and left not) enough for a bird to rest thereon".

Das kann eine Zerstörung nie erreichen, dass kein Vogel mehr einen Standort findet, das erreicht nur eine Überschwemmung, eine Sintflut. Und damit ist auch die richtige Erklärung der Stelle schon angedeutet. Der Verfasser denkt an die Sintflutgeschichte und will sagen: er zerstörte das Land so, dass es war wie bei der grossen Flut, wo die aus der Arche ausgesandten Vögel keinen Standort (man-za-zu Gilg. XI. 149, 152) fanden. Der Vergleich mit den Wirkungen der grossen Flut muss ja so oft die Erfolge von Städte- und Länderverwüstungen illustrieren. Statt des üblichen allgemeinen abûbiš wird hier ein spezielles Bild zur Veranschaulichung gewählt, eine Episode aus dem Ganzen zitiert, in abgekürzter Form, die sich stilistisch freilich schecht genug in den Zusammenhang fügt.

An der entsprechenden Stelle der Omina steht (Z. 33 f.) mâtu ka-sal-la ana ip-ri u kar-me

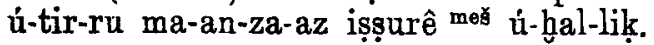

\section{Bemerkung zu OLZ. 1909, Sp. 11.}

Aram. הנם zu hebr.-aram. אנם ist sehr willkommen. Hebr. schon Est. 1, 8. Nachzutragen o Dieb und Räuber: TTer I $25_{25}=$ jBik I 63 ${ }_{34}$ Bk 67a jKid I 60a ${ }_{15}$. TMš I 86 ${ }_{20}$ Bk $115^{\text {b }}$ Meir b-Bar. p. $152 \mathrm{Nr} .1009$ Budapest. - TBK X $369_{15}$ GA Gaon Joel Müller 110. 158. Wilna 109. 246. - Traurige Erfahrungen verdichten sich zu dem Satze סת גוי אנם הוא Bk 45a (Levy, Kohut, BJacob irrig Snh 45 ${ }^{\mathrm{a}}$ ) Aram. in der- selben Formel אִ GA Gaon Hark 118 Joel Müller 186, Coronel 12. Tor. šel Rī̌ōnīm II 31 (Das. II 58 הגוים אנםים רם). Pl. Snh $33_{3}$ Hul $94^{\text {b }}$. jSuk IV 54 ${ }_{63}^{b}$ (so lies auch TPea II $19_{26}$ für אואסין). GA Gaon Joel Müller 285. Deriv.: אִָּ GA Gaon Wilna 246.

Immanuel Lïw.

\section{Besprechungen.}

A. T. Olmstead: Western Asia in the Days of Sargon of Assyria 722-705 B. C. (Cornell Studies in History and Political Science. Vol. II). New York, Holt and Company, 1908. Bespr. v. J. V. Práse b, Prag.

Im vorliegenden nett ausgestatteten Buche begrüssen wir einen im ganzen gelungenen Versuch, einen assyrischen Grosskönig als Mittelpunkt der gleichzeitigen GeschichteVorderasiens zumGegenstand einer fachmässig bearbeiteten Monographie vorzuführen. Dem Ref. liegt es fern, die Hindernisse geringzuschätzen, die sich bei dem momentanen Stand der Forschung einer solchen Arbeit entgegenstellen, und von diesem Gesichtspunkt aus ist auch die sachlich gut informierte Monographie zu beurteilen. Insbesondere ist die eigene Information des Verf. an Ort und Stelle, wo es heisst, keilinschriftlich überlieferte Stadt- und Landschaftsnamen in Syrien, Nordarabien und Kleinasien zu lokalisieren, hervorzuheben. In dieser Beziehung hat sich der junge amerikanische Forscher ein unbestreitbares Verdienst erworben. Seinen $Z$ weck hat demgemäss Olmstead folgendermassen ausgesprochen: the reconstruktion of the history on the basis of the topography, resulting in a number of new identifications, especially in Asia Minor.

Bevor wir ein Urteil über das vorliegende Werk Olmsteads abgeben, sehen wir uns vorher veranlasst, die Methode zu prüfen, die sich der Verf. in der Erörterung seiner Quellen zu eigen macht. In dieser Beziehung hat ihm bereits vor mehr als zwanzig Jahren in grossenteils abschliessender Weise $H$. Winckler vorgearbeitet. Seitdem sind zwar zahlreiche neue Dokumente keilinschriftlicher Art bekannt gemacht worden, aber die einschlägigen Aufstellungen Wincklers wurden dadurch nicht rückgängig gemacht. Ref. hat in seinen deutsch geschriebenen Arbeiten und in seinem böhmischen Werk: „Dèjiny starověkých národủ východnich" öfters Gelegenheit gehabt diese Aufstellungen kritisch zu prüfen, aber nur in wenigen Fällen hat er sich veraulasst gefunden von denselben abzuweichen. Die Stellungnahme Olmstead's zu Winckler muss daher als Prüfstein bei der Beurteilung seiner Monographie 